\title{
BMJ Open Physical activity for people with chronic kidney disease: an international survey of nephrologist practice patterns and research priorities
}

Airis Astiani Taryana, ${ }^{1}$ Rathika Krishnasamy, ${ }^{1,2}$ Clara Bohm, ${ }^{3,4}$ Suetonia C Palmer, ${ }^{5}$ Natasha Wiebe, ${ }^{6}$ Neil Boudville, ${ }^{7}$ Jennifer MacRae, ${ }^{8,9}$ Jeff Scott Coombes, ${ }^{10}$ Carmel Hawley, ${ }^{11}$ Nicole Isbel, ${ }^{11}$ Stephanie Thompson (iD ${ }^{12}$

To cite: Taryana AA, Krishnasamy R, Bohm C, et al. Physical activity for people with chronic kidney disease: an international survey of nephrologist practice patterns and research priorities. BMJ Open 2019;9:e032322. doi:10.1136/ bmjopen-2019-032322

- Prepublication history and additional material for this paper are available online. To view these files, please visit the journal online (http://dx.doi. org/10.1136/bmjopen-2019032322).

Received 16 June 2019 Revised 25 November 2019 Accepted 27 November 2019

Check for updates

(C) Author(s) (or their employer(s)) 2019. Re-use permitted under CC BY-NC. No commercial re-use. See rights and permissions. Published by BMJ.

For numbered affiliations see end of article.

Correspondence to Dr Stephanie Thompson; th11@ualberta.ca

\section{ABSTRACT}

Objectives People with chronic kidney diseases (CKD) have identified exercise as a research priority. To inform the research agenda, we surveyed nephrologists on their practice patterns, available resources and research priorities for exercise and physical activity (PA) in CKD.

Design Cross-sectional international survey.

Setting and participants 19-item electronic survey was administered to practising nephrologists with publicly available email addresses in Canada $(n=354)$ and Australia and New Zealand (ANZ) and via newsletters for the Australian and New Zealand Society of Nephrology $(\mathrm{n}=598)$.

Outcomes Frequency and predictors of exercise and PA counselling in practice and research priorities.

Results 189 respondents (20\% response) completed the survey. Eighty-one per cent of ANZ and $42 \%$ of Canadian respondents reported that their renal programmes did not have any exercise programmes or resources. The most frequently reported barrier for exercise programme implementation was a lack of funding $(77 \%)$. Ninety per cent of respondents thought regular exercise provides 'health benefits' for all CKD stages; 59\% reported that exercise counselling was within the nephrologists' scope of practice and $47 \%$ reported 'frequently' or 'always' counselling patients. In multivariable analysis, female gender (OR 2.31; 95\% $\mathrm{Cl} 1.16$ to 4.58) and older age (OR 1.94 per age category increase; $95 \% \mathrm{Cl} 1.15$ to 3.26 ) were associated with exercise counselling. Out of 194 research priorities, 65 (34\%) were clinical outcomes (cardiovascular parameters) and $30 \%$ were patient-reported outcomes (quality of life).

Conclusions Most nephrologists consider exercise and PA counselling as within their scope of practice and beneficial but, due to competing priorities, do not regularly counsel patients. This suggests a need for the evaluation of effective and efficient counselling strategies and a role for the routine involvement of exercise specialists in kidney care. Cardiovascular parameters and quality of life were identified as important outcomes for future exercise trials.

\section{INTRODUCTION}

Exercise is associated with a range of health benefits in people with chronic kidney disease
Strengths and limitations of this study

- This study extends the finding of previous studies by highlighting the need to address barriers to exercise counselling beyond the perception of benefit, such as the effectiveness of physician counselling in the chronic kidney disease setting.

- To better understand participants' responses, we included free text options.

- Only nephrologists with publicly available email addresses were surveyed and may not be representative of the nephrologists from each of these countries.

- Interpretation is limited by participant self-selection and a low response rate.

- Data on exercise and physical activity counselling were self-reported.

(CKD). ${ }^{1}$ Randomised trials have reported that exercise improves physical function, exercise capacity and health-related quality of life. ${ }^{1-3}$ Despite these demonstrable benefits, physical activity (PA) levels in people with CKD are low, ${ }^{4}$ and the prevalence of sedentary behaviour is approximately twice that of the general population. ${ }^{67}$ In addition, the frequency of exercise and PA counselling among nephrologists has historically been low. $^{5}$

Across a range of medical specialties, including nephrology, numerous barriers to exercise and PA counselling for physicians have been identified. These barriers include low confidence, the lack of skills, support and time. ${ }^{568-10}$ However, physician counselling can play an influential role in improving patients' PA levels. ${ }^{11}$ This suggests that for nephrologists, the patient encounter may represent an opportunity to support increased PA levels. In addition, exercise and PA guidelines from professional bodies on cardiovascular health 
recommend that patients with chronic diseases may benefit from similar targets for PA as the general population. The Kidney Disease Outcomes Quality Initiative clinical practice guidelines recommend that 'all dialysis patients should be counseled and regularly encouraged by nephrology and dialysis staff to increase their level of physical activity' (Guideline 14.2). ${ }^{12}$

The role of exercise in the management of CKD has also been recognised as a top research priority for patients and their caregivers. ${ }^{13}{ }^{14}$ Given the importance of this topic for patients and the increase in the evidence base to support the benefits of exercise in CKD,${ }^{12}$ this study aimed to evaluate contemporary nephrologists' counselling practice patterns with respect to exercise and PA among patients with CKD, including factors associated with exercise counselling behaviour. As a secondary aim, this study sought to identify nephrologist research priorities in this area.

\section{MATERIALS AND METHODS \\ Survey development}

A survey exploring nephrologists' practice patterns with respect to exercise and PA among patients with CKD was developed with the input of nine nephrologists from the participating countries (Canada and Australia and New Zealand (ANZ)). Survey items were generated through discussions among nephrologists and the literature. ${ }^{5}$ The survey was pilot tested on three nephrologists $(\mathrm{CB}, \mathrm{JM}$, RK) for length, ease of understanding, transferability of questions among the participating countries and face validity. Changes to the survey as a result of piloting were minor and addressed differences in practice setting and location between countries as well as clarity of phrasing.

\section{Survey participants and administration}

Invitations to complete the survey were emailed to practising nephrologists with publicly available email addresses in Canada $(\mathrm{n}=354)$ and $\mathrm{ANZ}$ and were also advertised in the newsletters for members of the Australian and New Zealand Society of Nephrology (ANZSN) $(\mathrm{n}=598)$. We included these three countries as the practice patterns and care delivery that could influence physician behaviour and exercise resources, such as reimbursement models and scope of practice are known to be similar. The contact email explained the purpose of the study and contained a link to access the secure web-based survey. The email and survey were translated into French for francophone participants in Canada. Participants were surveyed electronically between October 2017 and April 2018. Non-responders received two follow-up reminder emails, 2 weeks apart. In ANZ, the survey was considered as an evaluation activity not requiring ethical approval, as defined in the National Health and Medical Research Council guidance document. ${ }^{15}$ The participant's decision to complete and return the survey was interpreted as an indication of consent to participate.

\section{Study questionnaire}

The final survey consisted of 19 questions related to PA and exercise practice patterns and resources (online supplementary appendix 1) and could be completed in approximately $10 \mathrm{~min}$. We collected data on the participant demographics, years in practice, practice setting and population, and exercise habits. Agreement with counselling statements was measured on a 5-point Likert scale (strongly agree, agree, neutral, disagree, strongly disagree). Participants were asked to identify exercise resources and exercise barriers for individuals with CKD at their centre and to report their counselling practices on exercise and PA. Free text options were available for listing barriers and explaining counselling practices. Participants scored statements related to the benefits and safety of exercise according to the stage of CKD and were asked for their opinion on whether there was evidence in the literature to support regular exercise prescription for all patients with CKD (all stages). To identify research priorities, participants were asked to submit research questions or list knowledge gaps in the role of exercise for individuals with CKD that should be the focus for future research studies.

\section{Data analysis}

Due to similarities in practice and care delivery between ANZ, survey responses from these two countries were combined and compared with those of Canadian respondents using Fisher's exact tests. All analyses were performed in Stata/MP V.15.1 (www.stata.com). Descriptive results are reported as counts and percentages. Missing responses were excluded from the calculation of percentages. Two survey questions, 'Do you give specific advice on how to increase their level of physical activity?' and 'How often do you ask patients about their levels of physical activity?' were used to divide respondents into a counselling group (if they answered 'always' or 'frequently') and a (referent) non-counselling group (if they answered 'occasionally', 'infrequently' or 'never'). Univariable and multivariable logistic regressions were used to determine which variables were associated with exercise counselling. The multivariable models were fitted using backward elimination, removing variables significant in the univariable model when the Wald $\mathrm{p} \geq 0.05$. Submitted research questions were reviewed, coded according to the topic and categorised, and the frequency of responses was counted.

\section{Patient and public involvement}

Patients and the public were not involved in this survey.

\section{RESULTS}

\section{Respondent characteristics}

Characteristics of survey respondents are listed in table 1 . All survey responses are shown in online supplementary appendix 2. There were 198 respondents, however, nine were excluded as they answered only one question; 
Table 1 Participant characteristics, $\mathrm{n}(\%)$

\begin{tabular}{|c|c|c|c|c|c|}
\hline Characteristic & All & Canada & ANZ & P value* & Missing \\
\hline Variable & 189 & $104(55.0)$ & $85(45.0)$ & & \\
\hline Age (years) & & & & 0.42 & $0(0)$ \\
\hline$<35$ & $14(7.4)$ & $5(4.8)$ & $9(10.6)$ & & \\
\hline $35-50$ & $119(63.0)$ & $65(62.5)$ & $54(63.5)$ & & \\
\hline $51-65$ & $48(25.4)$ & $29(27.9)$ & $19(22.4)$ & & \\
\hline$>65$ & $8(4.2)$ & $5(4.8)$ & $3(3.5)$ & & \\
\hline Female & $63(33.3)$ & $31(29.8)$ & $32(37.7)$ & 0.28 & $0(0)$ \\
\hline Years in practice & & & & 0.01 & $0(0)$ \\
\hline $0-5$ & $31(16.4)$ & $9(8.7)$ & $22(25.9)$ & & \\
\hline $6-10$ & $43(22.8)$ & $26(25.0)$ & $17(20.0)$ & & \\
\hline $11-15$ & $45(23.8)$ & $27(26.0)$ & $18(21.2)$ & & \\
\hline $16-20$ & $34(18.0)$ & $24(23.1)$ & $10(11.8)$ & & \\
\hline$>20$ & $36(19.1)$ & $18(17.3)$ & $18(21.2)$ & & \\
\hline Setting & & & & - & $0(0)$ \\
\hline Academic & - & $77(74.0)$ & - & & \\
\hline Community & & $14(13.5)$ & & & \\
\hline Mix of academic/community & & $13(12.5)$ & & & \\
\hline Public & - & - & $76(89.4)$ & & \\
\hline Private & & & $3(3.5)$ & & \\
\hline Mix of public/private & & & $6(7.1)$ & & \\
\hline Population & & & & 0.50 & $0(0)$ \\
\hline Primarily adult & $179(94.7)$ & $98(94.2)$ & $81(95.3)$ & & \\
\hline Primarily paediatric & $9(4.8)$ & $6(5.8)$ & $3(3.5)$ & & \\
\hline Both adult and paediatric & $1(0.5)$ & $0(0.0)$ & $1(1.2)$ & & \\
\hline Personal physical activity & & & & 0.21 & $0(0)$ \\
\hline Almost never or never & $7(3.7)$ & $5(4.8)$ & $2(2.4)$ & & \\
\hline Less than once a week & $8(4.2)$ & $6(5.8)$ & $2(2.4)$ & & \\
\hline Once a week & $20(10.6)$ & $12(11.5)$ & $8(9.4)$ & & \\
\hline 2-3 times/week & 63 (33.3) & $27(25.0)$ & $36(42.4)$ & & \\
\hline 4-5 times/week & $52(27.5)$ & $29(27.9)$ & $23(27.1)$ & & \\
\hline Daily or almost daily & $39(20.6)$ & $25(24.0)$ & $14(16.5)$ & & \\
\hline
\end{tabular}

*Fisher's exact tests comparing Canada to ANZ. ANZ, Australia and New Zealand.

therefore, 189 survey responses were included in this report: $104(55 \%)$ from Canada and $85(45 \%)$ were from ANZ. The overall response rate was 20\%. Sixtythree per cent were between the ages of 35 and 50, 25\% were between 51 and 65 and $7 \%$ were under 35 . Thirtythree per cent were female. Most Canadian (74\%) and ANZ respondents $(89 \%)$ practised in academic centres and public hospitals, respectively. Approximately 5\% of respondents provided care for paediatric patients. Overall, 33\% reported exercising or doing PA between two and three times per week and 28\% reported exercise activity four to five times per week.

\section{Resources and barriers}

Thirteen per cent of respondents did not know if exercise resources were provided by their renal programme (eg, counselling from an exercise professional, access to classes or equipment) and $61 \%$ reported that their centres did not offer any exercise resources for individuals with CKD at any stage. Notably, $81 \%$ of ANZ and $42 \%$ of Canadian respondents reported that their centres did not have any renal exercise programmes. In Canada, in-centre haemodialysis exercise programmes were the most commonly reported exercise resource for renal patients. Less than $5 \%$ of ANZ respondents reported the availability of exercise resources for CKD at any stage. 


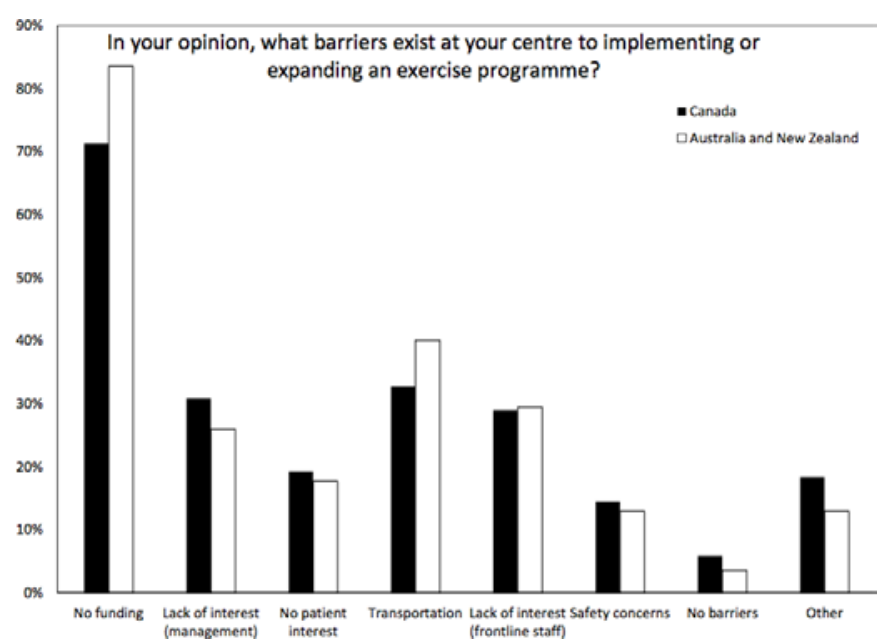

Figure 1 Barriers to exercise programme implementation and expansion.

Barriers to exercise programme implementation are shown in figure 1. Overall, the most frequently reported barrier for exercise programme implementation and development was a lack of funding (77\%) followed by difficulty with transportation (getting participants to the exercise programme) (36\%). Lack of personnel, leadership and expertise were additional frequently mentioned barriers.

\section{Nephrologist perception and practice patterns}

Nephrologist opinions and practice patterns related to exercise and PA counselling are shown in figure 2. Overall, $59 \%$ thought that nephrologists should take ownership in providing exercise counselling and resources to individuals with CKD and $71 \%$ thought that it is the kinesiologists/exercise physiologists/physiotherapists' job. Only $37 \%$ and $53 \%$ thought that primary care physicians and renal allied health professionals, respectively, should take ownership in providing exercise counselling and resources to patients with CKD. Nearly all respondents $(90 \%)$ agreed that regular exercise provides 'health benefits' for all CKD stages. Additionally, 71\% reported 'frequently' or 'always' asking patients about their PA levels and $47 \%$ 'frequently' or 'always' providing patients with specific advice on exercise. Overall, $81 \%$ agreed or strongly agreed that exercise can help with dialysisrelated symptoms. The most common reasons for 'never' or 'infrequently' assessing patients' PA levels or counselling patients on exercise were a perception that the time would be better spent on other parameters, not knowing the specific exercise prescription and a perception that patients would not follow exercise suggestions from nephrologists because they were not experts in exercise.

Overall, $38 \%$ of respondents agreed that sufficient evidence exists to support regular exercise prescription and participation for individuals with CKD and 35\% expressed that they did not know whether there is the evidence base to support this. In univariate analysis, respondent's gender, personal PA level and confidence level to recommend PA were associated with counselling behaviour. In multivariable analysis, we found that female respondents (OR 2.31; 95\% CI 1.16 to 4.58), older respondents (OR 1.94 per age category increase; $95 \%$ CI 1.15 to 3.26) and confidence level in the ability to recommend exercise (OR 2.63 per category increase; 95\% CI 1.79 to 3.86) were associated with counselling. Nephrologists' self-reported PA was associated with asking patients about their PA levels (OR 1.54 per PA category increase; 95\% CI 1.15 to 2.08 ) but not with providing specific advice on how to increase PA. The other items were dropped from the multivariable model, as they were not significant, including agreement with a sufficient evidence base for exercise in CKD. There were no significant differences in counselling behaviour between countries.

\section{Research priorities}

Forty per cent of respondents submitted at least one research question related to the role of exercise for individuals with CKD. A total of 194 research questions were submitted. The research priorities were coded according to the topic and inductively categorised into five broad research topics: clinical outcomes, patient-reported outcomes, exercise prescription, exercise programme implementation and feasibility, and functional outcomes (figure 3). Out of 194 topics, 65 (34\%) were clinical outcomes, 59 $(30 \%)$ were patient-reported outcomes, 26 (13\%) were exercise programme implementation and feasibility, 24 (12\%) were exercise prescription and $10(5 \%)$ were functional outcomes. Overall, the impact of exercise on clinical outcomes as a future research priority was mentioned most frequently (cardiovascular parameters $(22 \%)$ and CKD progression $(15 \%)$ ), followed by patient-reported outcomes (quality of life $(27 \%)$, symptoms $(25 \%)$ and mental health $(25 \%))$. The impact of exercise on mortality was not frequently mentioned as a research priority (14\%). Under the category of programme implementation and feasibility, patient motivation (19\%) and barriers to interest (19\%) were identified as important research questions. The type of exercise $(50 \%)$ was recognised to be the most important research question within the exercise prescription category. Specifically, the type of exercise that is safe and beneficial for individuals on different dialysis modalities, followed by the type of exercise for patients with advanced CKD, the type of exercise for CKD patients with chronic pain and the type of exercise that is 'most beneficial'. The specific modality or stage of CKD was specified for only the minority of submitted questions and most commonly, the topic was prioritised as important 'for all stages of CKD'.

\section{DISCUSSION}

This study aimed to examine contemporary nephrologists' perceptions and practice patterns regarding exercise and PA counselling for people with CKD and identify resources, barriers and research priorities. We found that although nearly all nephrologists surveyed (90\%) agreed that regular exercise provides 'health benefits' for all CKD stages and 59\% believed that nephrologists 

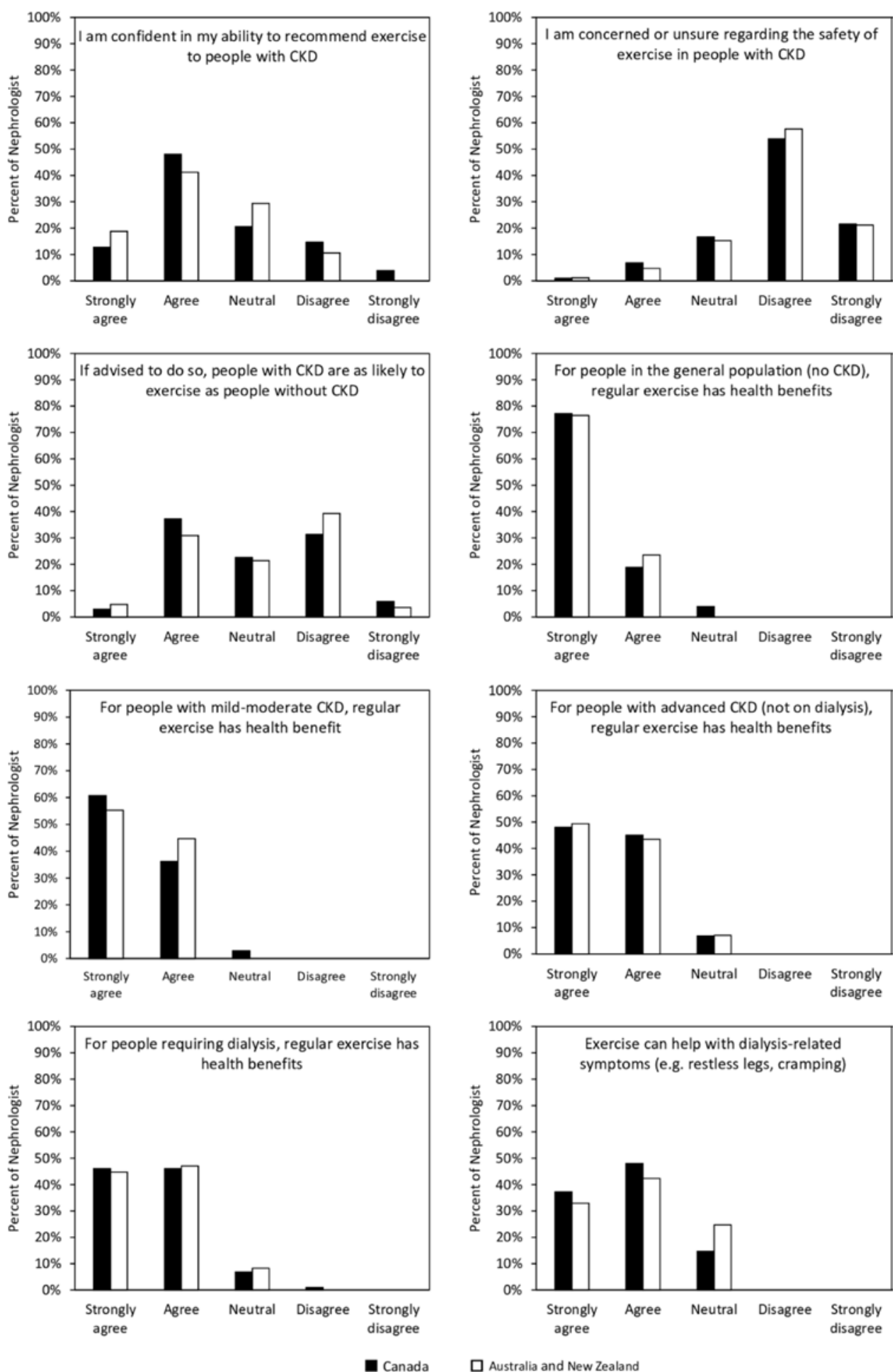

Figure 2 Nephrologist perception and practice patterns. CKD, chronic kidney disease.

should take ownership in providing exercise counselling and resources, only $47 \%$ reported 'always' or 'frequently' counselling patients to increase PA levels in practice. This is despite the finding that $81 \%$ of respondents agreed that exercise could help with dialysis-related symptoms.

The gap between agreeing with the benefits of PA and promoting $\mathrm{PA}$ in practice is in keeping with a survey of renal health professionals by Greenwood $e t a l^{4}$ In their study, Greenwood et al found the majority (91\%) of renal health professionals believed that exercise and PA are an important part of CKD management but only $59 \%$ asked and counselled patients with CKD about PA. ${ }^{4}$ This suggests that the trends and practice patterns have not changed and underscores the importance of addressing the specific barriers to exercise counselling beyond the perception of benefits. Consistent with previous studies, lack of time and the perception that PA was less important than other parameters were common barriers 


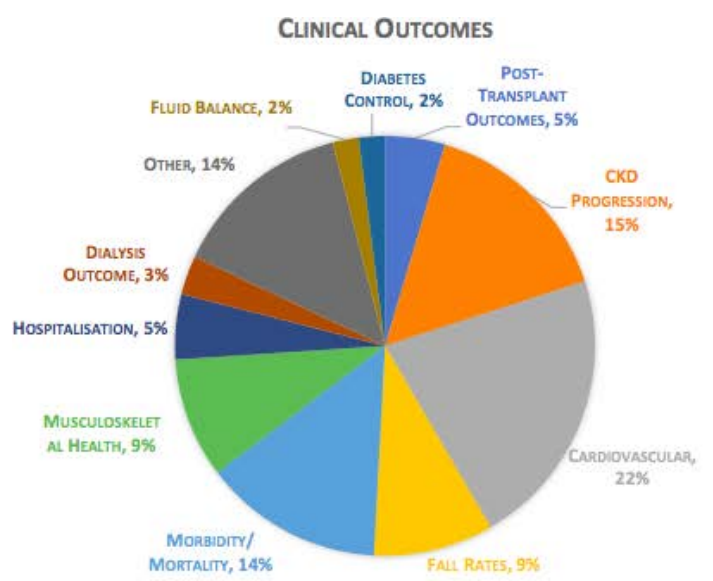

Functional OUTCOMES

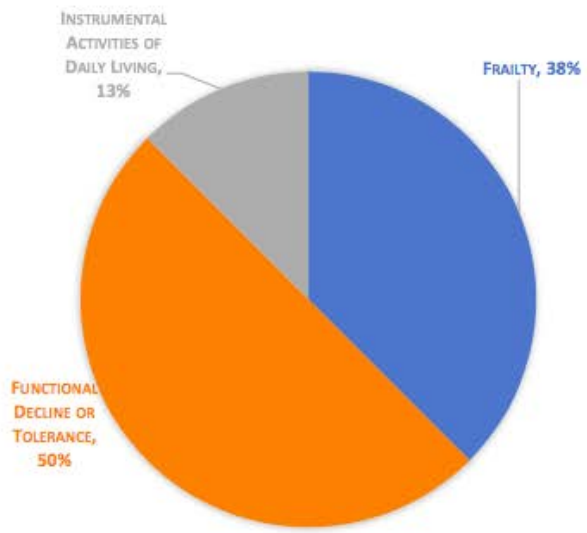

Patient-Reported

OUTCOMES

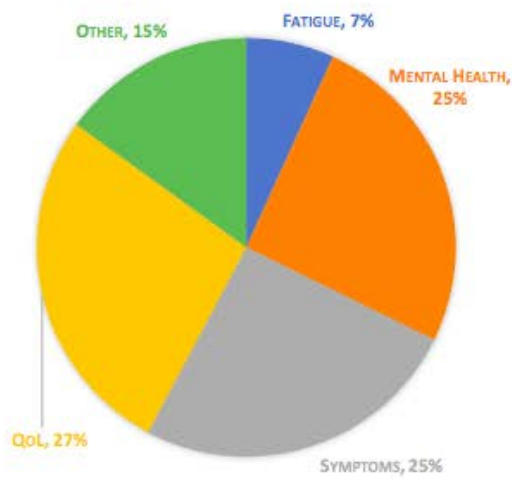

EXERCISE PRESCRIPTION

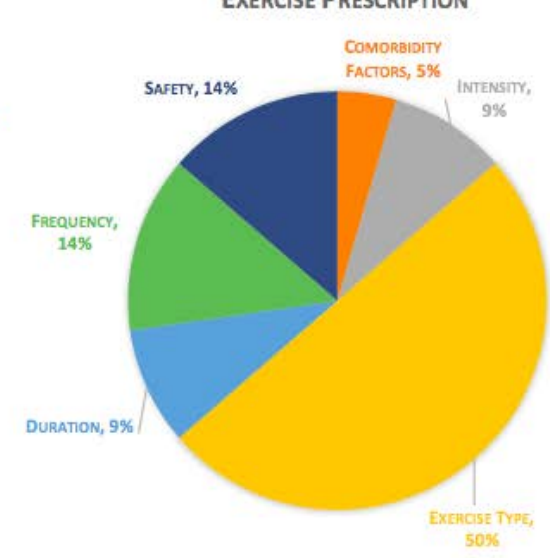

to counselling among nephrologists. ${ }^{5} 16$ In one study, nurses and nephrologists' negative perceptions towards counselling were associated with lower self-reported PA in haemodialysis patients. ${ }^{17}$ We identified additional barriers, which include the perception that patients would not exercise if advised to do so and that counselling would be less effective because nephrologists were not experts in exercise. These findings speak to the need for a greater awareness among nephrologists on the potential impact of exercise counselling and on how to counsel effectively. Although there is a need for more research in CKD on the latter, the type of counselling that has been effective in other populations with chronic conditions could be applied to most individuals with mild to moderate CKD. For example, a specific exercise prescription and goal setting increased exercise adherence in patients with heart failure. ${ }^{18}$ Employing individual counselling and interactive elements, such as audit and feedback systems, improved PA levels in patients with cancer undergoing treatments. ${ }^{19}$ It is also important to note that a single exercise counselling session is unlikely to improve PA in individuals with CKD. ${ }^{20}$ Therefore, in order to change behaviour, there is a need for ongoing support and motivation from dedicated exercise professionals. Along these same lines, exercise professionals have the expertise to inform patients on the specific exercise prescription.

Only $38 \%$ of respondents agreed that there was a sufficient evidence base to recommend exercise, while $35 \%$ expressed that they did not know the literature. This finding appears incongruent with the strong agreement regarding the benefits of exercise but underscores the importance of understanding the difference between the general perception that exercise is 'good' versus prescribing it as a therapy to treat a disease-related condition. Although there are expert recommendations on how to prescribe exercise in this population, ${ }^{21}$ there is a lack of evidence from clinical trials on exercise prescription to address symptoms that are both relevant to renal care and important to patients, such as restless legs syndrome and fatigue.

Physicians' age, gender and confidence level to recommend exercise were found to be important predictors of counselling behaviour. This finding is consistent with a 2010 survey on nephrologists' counselling practices by Delgado and Johansen ${ }^{5}$ where older nephrologists were more likely to ask and counsel patients on exercise compared with younger nephrologists. In contrast, we found that although nephrologists' PA levels were 
associated with higher odds of asking about PA, there was no association with providing specific advice on how to become more active. As providing specific advice on how to increase PA levels takes time and expertise, this difference is not surprising.

An additional aim of this study was to identify the research priorities with respect to exercise in CKD. We found that the questions that nephrologists most commonly identified as important were generally consistent with those identified by non-dialysis CKD and dialysis patients, namely the effect of exercise on the progression of CKD and the effect of exercise on dialysis patients' health, respectively. ${ }^{13}{ }^{14}$ These questions underscore patient and physician interest in examining the role of exercise as a therapeutic tool to manage both clinical and patient-reported outcomes. In our survey, the impact of exercise on patient-reported outcomes such as quality of life, symptoms and mental health was frequently mentioned as a research priority for CKD at all stages, which is timely given the recent focus on the importance of patient-reported outcomes and the increasing awareness of exercise as an important issue for patients and their caregivers. ${ }^{1422} 23$ Additionally, nephrologists identified research on specific types of exercise prescription for individuals with CKD as a priority, highlighting the need for research comparing different exercise prescriptions in well-defined CKD populations.

This study has several limitations that may have affected the generalisability of the results. First, we only surveyed nephrologists with publicly available email addresses in Canada and ANZ and practising nephrologists who were members of the ANZSN. Second, nephrologists with a personal or professional interest in exercise may have been more likely to participate and non-response bias could have influenced the results. Third, the response rate was low; however, a $20 \%$ response rate is comparable to other published surveys on this topic as well as response rates from online surveys with clinicians in general. ${ }^{42}$ Lastly, there was a larger response rate from Canada $(55 \%)$ versus ANZ (45\%) and more men than women responded, which may have influenced the findings.

\section{CONCLUSION}

Most nephrologists consider exercise and PA counselling as within their scope of practice and conferring health benefits in CKD, but do not regularly counsel patients. The infrequency of routine counselling may relate to the complexity of practice and competing priorities. Therefore, there needs to be a restructuring of nephrology care to support exercise and PA uptake, such as with the routine involvement of an exercise professional in multidisciplinary kidney care. Given the potential for the physician's advice to positively influence exercise adoption and the awareness of the importance of exercise counselling skills, more research on how and what to recommend for exercise and PA in people with CKD is needed.
Author affiliations

${ }^{1}$ Faculty of Medicine, The University of Queensland, Brisbane, Queensland, Australia ${ }^{2}$ Australasian Kidney Trials Network, The University of Queensland, Brisbane, Queensland, Australia

${ }^{3}$ Department of Internal Medicine, Max Rady College of Medicine, University of Manitoba, Winnipeg, Manitoba, Canada

${ }^{4}$ Chronic Disease Innovation Centre, Seven Oaks General Hospital, Winnipeg, Manitoba, Canada

${ }^{5}$ Department of Medicine, University of Otago Christchurch, Christchurch, New Zealand

${ }^{6}$ Department of Medicine, University of Alberta, Edmonton, Alberta, Canada ${ }^{7}$ Medical School, University of Western Australia, Perth, Western Australia, Australia ${ }^{8}$ Department of Medicine, Cumming School of Medicine, University of Calgary, Calgary, Alberta, Canada

${ }^{9}$ Department of Cardiac Sciences, Cumming School of Medicine, University of Calgary, Calgary, Alberta, Canada

${ }^{10}$ School of Human Movement and Nutrition Sciences, The University of Queensland, Brisbane, Queensland, Australia

${ }^{11}$ Department of Nephrology, Princess Alexandra Hospital, South Brisbane, Queensland, Australia

${ }^{12}$ Department of Medicine, University of Alberta, Edmonton, Alberta, Canada

Twitter Stephanie Thompson @StephanieTh11

Acknowledgements We thank Ghenette Houston for support with organisation and conduct of the survey.

Contributors ST, JM, CB and RK were responsible for the inception and design of the project and contributed to manuscript development. AAT contributed to the analysis and prepared the manuscript. NW performed the analysis and provided methodological input. SP, NB, CH, JSC and NI contributed methodological input and to the development of the manuscript.

Funding The authors have not declared a specific grant for this research from any funding agency in the public, commercial or not-for-profit sectors.

Competing interests None declared.

Patient consent for publication Not required.

Provenance and peer review Not commissioned; externally peer reviewed.

Data availability statement Data are available upon reasonable request.

Open access This is an open access article distributed in accordance with the Creative Commons Attribution Non Commercial (CC BY-NC 4.0) license, which permits others to distribute, remix, adapt, build upon this work non-commercially, and license their derivative works on different terms, provided the original work is properly cited, appropriate credit is given, any changes made indicated, and the use is non-commercial. See: http://creativecommons.org/licenses/by-nc/4.0/.

ORCID iD

Stephanie Thompson http://orcid.org/0000-0003-3109-6837

\section{REFERENCES}

1 Heiwe S, Jacobson SH. Exercise training in adults with CKD: a systematic review and meta-analysis. Am J Kidney Dis 2014;64:383-93.

2 Heiwe S, Jacobson SH. Exercise training for adults with chronic kidney disease. Cochrane Database Syst Rev 2011;19.

3 Smart NA, Titus TT. Outcomes of early versus late nephrology referral in chronic kidney disease: a systematic review. Am J Med 2011;124:1073-80.

4 Greenwood SA, Koufaki P, Rush R, et al. Exercise counselling practices for patients with chronic kidney disease in the UK: a renal multidisciplinary team perspective. Nephron Clin Pract 2014;128:67-72.

5 Delgado C, Johansen KL. Deficient counseling on physical activity among nephrologists. Nephron Clin Pract 2010;116:c330-6.

6 Beddhu S, Wei G, Marcus RL, et al. Light-Intensity physical activities and mortality in the United States general population and CKD subpopulation. Clin J Am Soc Nephrol 2015;10:1145-53.

7 Glavinovic T, Ferguson T, Komenda P, et al. CKD and Sedentary Time: Results From the Canadian Health Measures Survey. Am J Kidney Dis 2018;72:529-37. 
8 Kennedy MF, Meeuwisse WH. Exercise counselling by family physicians in Canada. Prev Med 2003;37:226-32.

9 Nadler M, Bainbridge D, Tomasone J, et al. Oncology care provider perspectives on exercise promotion in people with cancer: an examination of knowledge, practices, barriers, and facilitators. Support Care Cancer 2017;25:2297-304.

10 Pang A, Lingham S, Zhao W, et al. Physician practice patterns and barriers to counselling on physical activity in solid organ transplant recipients. Ann Transplant 2018;23:345-59.

11 Greenlund KJ, Giles WH, Keenan NL, et al. Physician advice, patient actions, and health-related quality of life in secondary prevention of stroke through diet and exercise. Stroke 2002;33:565-71.

12 Workgroup KD. K/DOQI clinical practice guidelines for cardiovascular disease in dialysis patients. Am J Kidney Dis 2005;45:S1-153.

13 Hemmelgarn BR, Pannu N, Ahmed SB, et al. Determining the research priorities for patients with chronic kidney disease not on dialysis. Nephrol Dial Transplant 2017;32:847-54.

14 Manns B, Hemmelgarn B, Lillie E, et al. Setting research priorities for patients on or nearing dialysis. Clin J Am Soc Nephrol 2014;9:1813-21.

15 NHMRC. Ethical considerations in quality assurance and evaluation activities. Australia: National Health and Medical Research Council, 2014.

16 Johansen KL, Sakkas GK, Doyle J, et al. Exercise counseling practices among nephrologists caring for patients on dialysis. Am J Kidney Dis 2003;41:171-8.
17 Regolisti G, Maggiore U, Sabatino A, et al. Interaction of healthcare staff's attitude with barriers to physical activity in hemodialysis patients: a quantitative assessment. PLOS One 2018;13:e0196313.

18 Tierney S, Mamas M, Woods S, et al. What strategies are effective for exercise adherence in heart failure? A systematic review of controlled studies. Heart Fail Rev 2012;17:107-15.

19 IJsbrandy C, Ottevanger PB, Tsekou Diogeni M, et al. Review: effectiveness of implementation strategies to increase physical activity uptake during and after cancer treatment. Crit Rev Oncol Hematol 2018;122:157-63.

20 Bohm CJ, Storsley LJ, Hiebert BM, et al. Impact of exercise counseling on physical function in chronic kidney disease: an observational study. Can J Kidney Health Dis 2018;5.

21 Koufaki P, Greenwood S, Painter P, et al. The bases expert statement on exercise therapy for people with chronic kidney disease. J Sports Sci 2015;33:1902-7.

22 Tong A, Winkelmayer WC, Wheeler DC, et al. Nephrologists' perspectives on defining and applying patient-centered outcomes in hemodialysis. Clin J Am Soc Nephrol 2017;12:454-66.

23 Flythe JE, Hilliard T, Lumby E, et al. Fostering innovation in symptom management among hemodialysis patients: paths forward for insomnia, muscle cramps, and fatigue. Clin J Am Soc Nephrol 2019;14:150-60.

24 Dykema J, Jones NR, Piché T, et al. Surveying clinicians by web: current issues in design and administration. Eval Health Prof 2013;36:352-81. 